\title{
Modernisation des anciens canaux d'adduction
}

\author{
par H. Pochet et R.J. Chauvet \\ Société du Canal de Provence, \\ Le Tholonet
}

La modernisation d'un canal d'adduction d'eau peut avoir deux objectifs principaux :

- faciliter l'exploitation des ouvrages pour alléger les frais de gestion;

- reconvertir le canal pour l'adapter à de nouvelles conditions de distribution et pour réduire, en particulier, lo gaspillage de l'eau.

\section{Modernisation d'un canal pour de meilleures conditions d'exploitation}

De nombreux canaux anciens sont équipés de déversoirs transversaux, réalisés le plus souvent à l'aide de batardeaux amovibles, afin de maintenir un plan d'eau à une cote suffisante pour alimenter les prises d'eau.

La longueur déversante était relativement faible, le réglage du niveau est très approximatif et il doit être rétabli, à chaque changement notable du débit, en modifiant la hauteur du batardeau.

D'autre part, les prises d'eau sur ces anciens canaux comprennent, généralement, des martelières réglant l'ouverture d'un orifice qui débouche dans un petit bassin de dissipation comportant un déversoir de mesure de débit. Le garde-canal procède par tâtonnement pour régler le débit et il est obligé d'effectuer des réajustements lorsque le plan d'eau varie dans le canal.

Toutes ces manœuvres de batardeaux et de martelières représentent un travail continuel et pénible qui nécessite un personnel nombreux.

Le souci d'une meilleure rentabilité des ouvrages de transport conduit donc à rechercher des solutions pour alléger les frais d'exploitation en assurant l'automaticité des réglages des niveaux et des débits des prises d'eau.

a) LE RÉGLAGE DES NIVEAUX dans le canal peut être amélioré en construisant des déversoirs fixes avec un grand développement en crête (déversoirs obliques ou déversoirs en «bec de canard $»$ ), mais la solution moderne est d'utiliser des vannes à niveau amont constant (vanne AMIL) qui assurent un réglage automatique et précis du niveau. Les avantages essentiels de ces vannes, par rapport aux déversoirs, sont essentiellement qu'elles ne créent qu'une faible perte de charge et qu'elles n'entraînent aucun risque de dépôt puisque l'écoulement se fait sous le tablier.

b) LE RÉGlage DES DÉBITS des prises d'eau sora rendu extrêmement simple en équipant celles-ci de modules à masque. Il suffit d'ouvrir le nombre de vannettes correspondant au débit que l'on désire envoyer. Il n'y a plus de tâtonnement et d'attente avec, en outre, la certitude que ce débit restera stable si la prise d'eau n'est pas trop éloignée de l'ouvrage de réglage de niveau.

Pour ne pas multiplier les ouvrages de réglage de niveau dans le canal principal, on peut, moyennant une perte de charge un peu plus forte, placer au départ de la prise une vanne à niveau aval constant (vanne AVIO) précédant la batterie de modules.

\section{Modernisation d'un canal pour l'adapter à de nouvelles conditions de distribution de l'eau}

Une évolution importante a marqué, pendant ces deux dernières décennies, le mode de distribution de l'eau. En effet, l'irrigation par aspersion avec distribution de l'eau 
«à la demande » remplace, de plus en plus, l'irrigation par ruissellement avec distribution de l'eau au «tour d'arrosage».

Or, le passage à une distribution de l'eau «à la demande» dans un canal équipé pour une distribution au «tour d'arrosage » pose des problèmes de régulation très compliqués et conduit, inévitablement, à des pertes d'eau massives par les décharges du canal, à cause des variations importantes et aléatoires de cette demande.

La notion de l'économie de l'eau, qui est primordiale dans les régions souffrant de pénurie et qui est de plus en plus prise en considération, même dans les zones disposant de ressources suffisantes, afin de pouvoir les utiliser au mieux, conduit ainsi à rechercher les moyens propres à réduire les gaspillages d'eau et à assurer la sécurité du transport dans ces canaux anciens.

La solution, pour assurer le contrôle du transport de l'eau, est d'équiper le canal avec un nouveau mode de régulation.

Les modes de régulation modernes, qui peuvent en principe être mis en compétition pour l'équipement des canaux de transport anciens, sont: la «commande par l'aval», la régulation à «niveaux associés» et la «régulation dynamique ».

La COMMANDE PAR L'AVAL, classique, est un système bien connu qui a fait ses preuves et qui peut, pour des projets de canaux nouvoaux, répondre parfaitement au but recherché, c'est-à-dire l'automatisme intégral du transport.

Mais ce mode de régulation ne peut s'appliquer qu'à des canaux à faible pente car, pour des canaux à pente relativement importante (supérieure à $0,50 \mathrm{~m} / \mathrm{km}$ pour fixer les idées), comme cela est souvent le cas pour des canaux anciens, les vannes seraient trop rapprochées les unes des autres et on arriverait à une impossibilité sur le plan de la stabilité du fonctionnement hydraulique.

Un autre inconvénient majeur de ce système de régulation est que les berges du canal doivent être horizontales entre deux vannes, ce qui nécessite donc la construction de surhausses par des apports de remblais ou des reprises de bétonnage souvent difficilement réalisables.

La Régulatron a nIVEauX associés, dont le système BIVAL est un type récemment breveté, est aussi une régulation en commande par l'aval mais qui, par rapport au système classique, permet de réduire les surhausses des berges puisque celles-ci ne doivent être horizontales que sur la partie aval de chaque bief.

Elle nécessite, par contre, des capteurs de niveau, une ligne de télétransmission le long du canal et des régulateurs commandant les servo-mécanismes des vannes qui rendent le système plus fragile.

Pour assurer la sécurité du transport de l'eau, il semble indispensable d'établir une centralisation des contrôles de fonctionnement par un réseau de télétransmission relié à un dispatching. Avec ces aménagements complémentaires, on disposerait alors de l'infrastructure nécessaire à une régulation dynamique qui présente de meilleurs avantages.

LA RÉGULATION DYNAMiQue. Nous rappellerons d'abord brièvement le principe de fonctionnement de ce nouveau mode de régulation:

Il s'agit d'un système qui, à l'aide d'un équipement de télétransmission et d'un calculateur industriel, assure automatiquement les différents réglages des vannes le long du canal, en fonction des besoins en eau à satisfaire.
L'ensemble du canal est géré comme un grand réservoir par le calculateur qui, recevant les différentes données sur l'état du canal (niveau de remplissage des biefs, position des vannes) et sur les consommations des diverses prises d'eau, les exploite pour faire le bilan des apports, des prélèvements et des réserves de chaque bief; il détermine ensuite les débits nécessaires en tête de chacun d'eux, pour maintenir ou rétablir l'équilibre dans les instants qui vont suivre en fonction des prévisions sur l'évolution de la consommation; il élabore, enfin, les nouvelles positions des vannes et les pilote par télécommande jusqu'à ce qu'elles aient atteint le degré d'ouverture nécessaire.

Pour la reconversion des canaux anciens, ce mode de régulation présente trois avantages économiques primordiaux :

$1^{\circ}$ il ne nécessite pas de surélévation des berges du canal;

$2^{\circ}$ il permet, le plus souvent, de réutiliser les systèmes de vannage existants qui devront simplement être complétés par une motorisation;

$3^{\circ}$ il est essentiellement évolutif, c'est-à-dire qu'il présente des degrés de sophistication très variés qui permettent de choisir le niveau de perfectionnement de l'aménagement en fonction de la valeur de l'eau économisée et des possibilités dinvestissement.

Pour la réalisation de plusieurs projets de modernisation de canaux anciens, la Société du Canal de Provence a adopté la régulation dynamique, comme nous le verrons dans les exemples d'aménagements suivants.

\section{Exemples d'aménagements}

\section{Extensions du canal du Verdon}

Les extensions du canal du Verdon comprennent trois branches. Ses deux branches principales sont la branche mère, de $11 \mathrm{~km}$ de longueur avec une portance de $6,5 \mathrm{~m}^{3} / \mathrm{s}$, et la branche de Gardanne, de $15 \mathrm{~km}$ de longueur et un débit de $4,5 \mathrm{~m}^{3} / \mathrm{s}$.

La reconversion de l'exploitation en commande par l'amont manuelle, de ces deux branches, s'est avérée nécessaire pour permettre une irrigation par aspersion à la demande et pour alléger les frais de gestion.

Une première étude a donc été faite pour aménager la branche mère et la branche de Gardanne, en vue d'une régulation en commande par l'aval. Elle a montré que l'équipement nécessiterait une quinzaine de vannes à niveati aval constant et des rehausses de berges entraînant une dépense prohibitive.

C'est alors que, progressivement, a été développée une télésurveillance de ces branches qui a évolué vers la mise au point du système de régulation dynamique, automatiquement et directement géré par un calculateur industriel I.B.M. 1800 placé au Centre Général d'Exploitation.

La régulation réutilise les vannes qui existaient sur l'ouvrage (vanne papillon au pied du barrage de Bimont. partiteur proportionnel des Sauvaires, vanne wagon de la chute des Figassons). Elles ont simplement été motorisées et équipées de télécommandes électriques gérées par le calculateur. 
SYSTEME ANCIEN

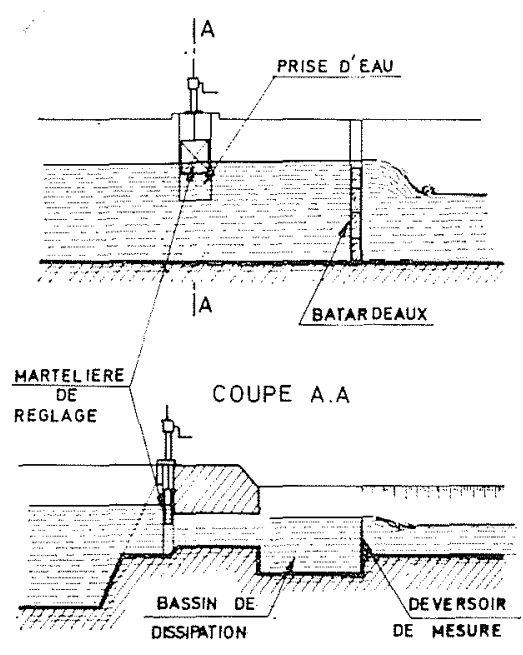

SYSTEME MODERNE

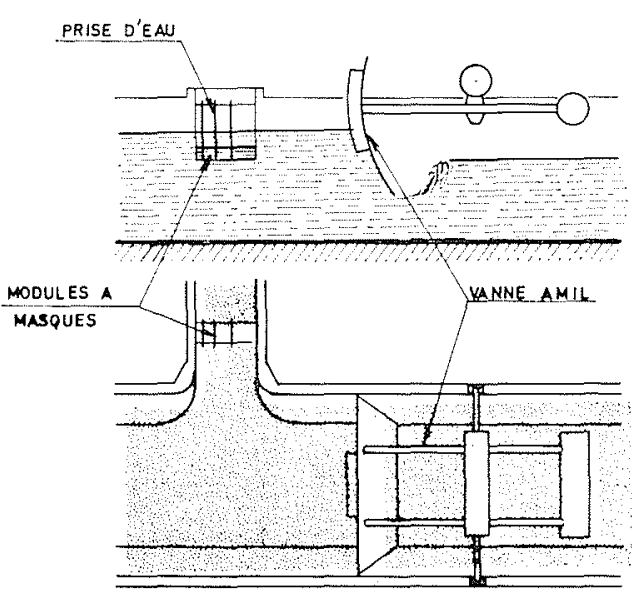

1/ Modernisation des prises d'eau.

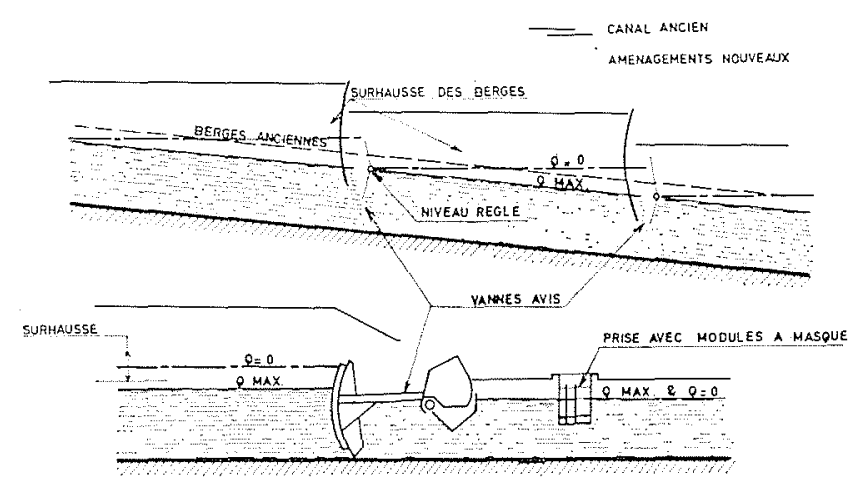

2/ Modernisation du canal par régulation en commande par l'aval.

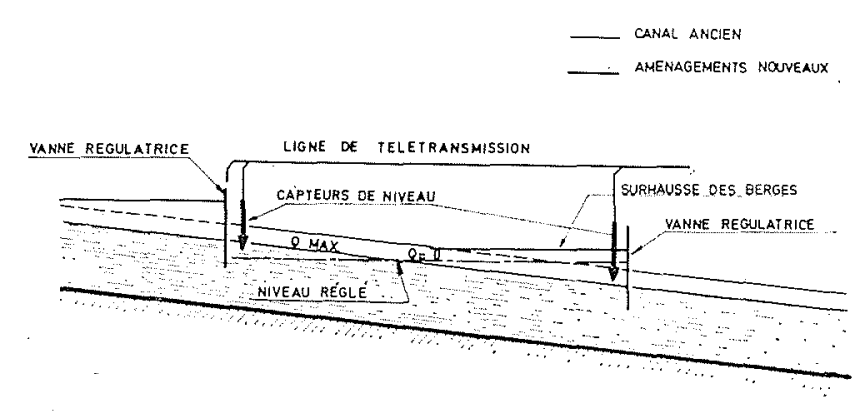

3/ Modernisation du canal par le Système Bival.

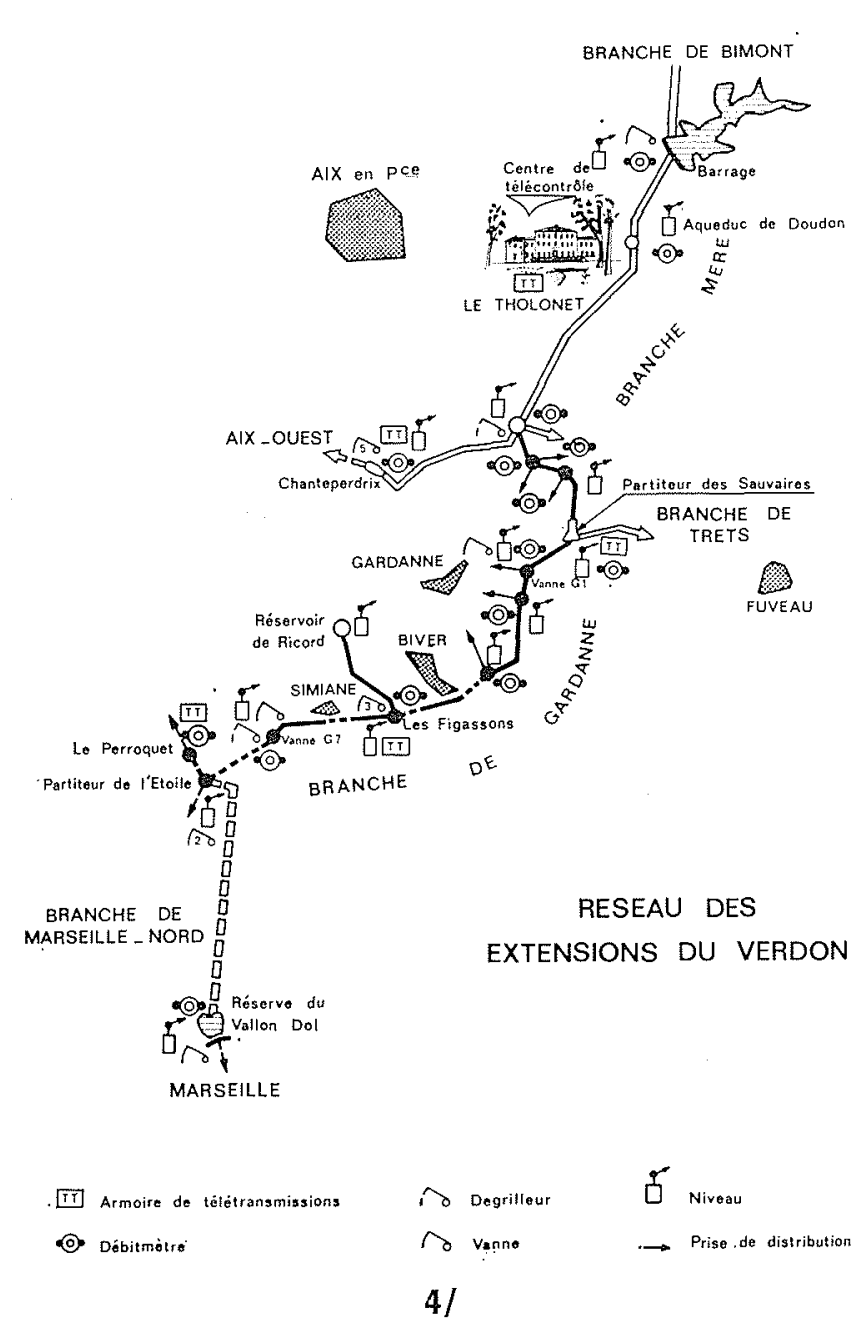


Neuf déversoirs en «bec de canard ont été construits dans le canal pour remplacer les déversoirs batardeaux: deux de ces ouvrages ont été équipés de vannes télécommandées pour la régulation des réserves de bief.

La régulation dynamique est en exploitation sur ces deux branches depuis la saison d'arrosage 1971, après une anné: de mise au point et d'essais préalables.

\section{Canal de Craponne}

Le canal de Craponne, long de $40 \mathrm{~km}$ avec une portance de $15 \mathrm{~m}^{3} / \mathrm{s}$, est un très vieux canal en terre, autrefois dérivé de la Durance, et actuellement réalimenté par le canal usinier de l'E.D.F.

Il est essentiellement affecté à l'irrigation par ruissellement de la Crau en basse Durance.

L'eau est distribuée au «tour d'arrosage» et le débit, réglé en tête du canal, est égal à la somme des droits d'eau; cependant, les agriculteurs ont tendance à ralentir les irrigations la nuit, ainsi que lors de la coupe des foins ou pendant les orages. De ce fait, le débit du canal est, à certaines heures, supérieur aux besoins et cela se traduit par des pertes d'eau qui atteignent annuellement 25 à $30 \mathrm{Mm}^{3}$ sur le canal principal, soit 15 à $18 \%$ du volume dérivé.

Une étude a été faite pour limiter ces pertes. Cette étude prévoit d'équiper le canal en régulation dynamique.

\section{L'équipement comprendra :}

- neuf mesures de niveau, placées dans les différents biets du canal, et ramenées par un réseau de télétransmission dans un dispatching central; celles-ci permettront de suivre l'évolution globale de la demande et du débit du canal;

- une télécommande des modules de tête qui assurera, à partir du dispatching, le réglage du débit en tête du canal;

- une réserve de $25000 \mathrm{~m}^{3}$, placée à mi-parcours, avec télécommande des vannes d'entrée et de sortie; cette réserve permettra, par remplissage ou déstockage, d'améliorer la correction des écarts entre la demande et le débit du canal.

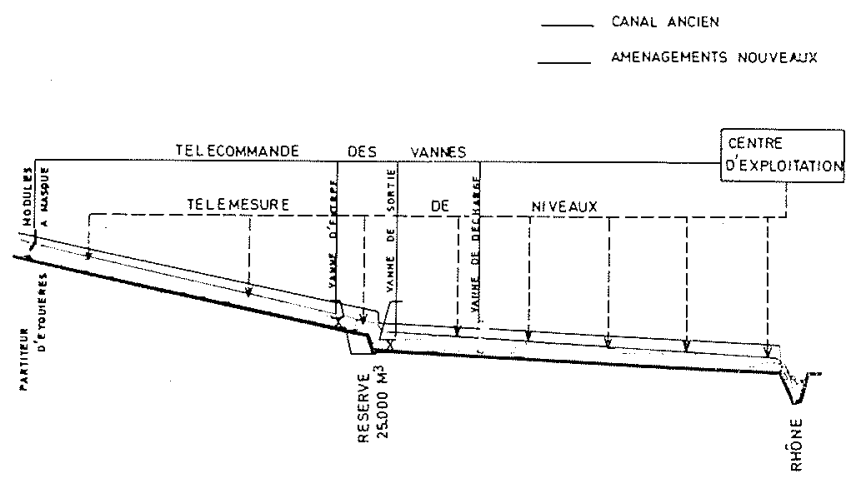

5/ Canal de Craponne. Projet de régulation.

\section{Adduction Mornos-Athènes}

Cette adduction a une longueur de $190 \mathrm{~km}$ et une portance de $23 \mathrm{~m}^{3} / \mathrm{s}$. Elle alimente, à partir du barrage de Mornos, la station de traitement des eaux d'Athènes. Elle comprend des canaux $(110 \mathrm{~km})$, des galeries $(70 \mathrm{~km})$ et des siphons $(10 \mathrm{~km})$.

Il ne s'agit pas, à proprement parler, d'un canal ancien, mais d'une adduction dont les travaux étaient déjà très avancés lorsque les études de régulation ont été entreprises.

Pour n'entraîner que le minimum de modifications aux ouvrages déjà réalisés ou en cours de construction, seule la régulation dynamique a pu être retenue.

Cette régulation a deux buts principaux:

- assurer la sécurité du fonctionnement indispensable, en raison de l'énorme masse d'eau $\left(2700000 \mathrm{~m}^{3}\right.$ à $Q$ : $23 \mathrm{~m}^{3} / \mathrm{s}$ ) contenue dans le canal en amont de la ville d'Athènes;

- permettre une modulation relativement rapide des débits à l'arrivée à la station de traitement; en effet, étant donné la grande longueur de l'ourvrage, il faut un délai de 24 heures pour qu'une modification du débit à la prise se répercute à la station de traitement.

La sécurité sera assurée :

- par des bRISE-Charges placés à la sortie des galeries pour les maintenir en charge à tous les régimes;

- PAR DES DÉverSoIRs BEC DE CANARD équipés de vannes télécommandées, qui retiendront un volume d'eau important dans les cuvettes.

LA MODULATION DES déBITS sera réglée par les mêmes ouvrages «bec de canard»: la manœuvre télécommandée des vannes de ces ouvrages modifiena le niveau de remplissage des biefs et donc modulera les débits à l'arrivée dans la station de traitement d'Athènes.

Un RÉseau de TÉlÉmesure des niveaux, des débits et des positions des vannes, assurera la surveillance continue du fonctionnement du canal; UN RÉSEAU DE TÉLÉCOMMANDE permettra d'effectuer les manœuvres de sécurité (c'est-à-dir'e la fermeture des vannes en amont de la zone éventuellement accidentée) et les manœulvres de modulation à partir du centre de contrôle situé à la station de traitement des eaux d'Athènes.

\section{Problèmes particuliers de Génie civil posés par la modernisation des anciens canaux d'adduction}

\section{Type d'intervention de Génie civil}

Il est évident que la modernisation d'un ancien canal ne présente d'intérêt que si cet ouvnage ne peut être plus économiquement remplacé par un canal neuf assurant un service au moins égal.

Cette modernisation entraîne le plus souvent:

- des réfections et réparations de gros entretien pour les parties les plus vétustes; 
- des travaux permettant d'améliorer les conditions d'utilisation de l'ouvrage: augmentation de la capacité de transport, adjonction de prises nouvelles, etc.

Les interventions de génie civil sont donc multiples et on peut les classer en trois grandes catégories.

\section{Première catégorie}

Elle comprend des constructions et modifications locales impliquées par l'adoption d'un type de régulation différent, par exemple :

- les suppressions d'anciens batardeaux manœuvrés à la main;

- la construction, dans le canal, de déversoirs en «bec de canard » et d'ouvrages de vannages nouveaux;

- les petites constructions et les adaptations destinéos aux organes de télémesure et tólécommande (abris d'organes de commande, passage de câbles, modifications éventuelles d'ouvrages existants);

- les rehausses des berges, nécessaires en cas d'adoption d'une régulation en commande par l'aval ou à niveaux associés.

On peut toutefois signaler que, parmi toutes ces modifications, les rehausses des berges, qui représentent en fait les seuls travaux importants, ne sont pas nécessaires si on équipe le canal d'une régulation dynamique.

\section{Deuxième catégorie}

Elle comprend les constructions et les modifications nécessaires pour améliorer l'exploitation et développer les possibilités d'utilisation de l'ouvrage.

C'est pour atteindre ce premier but que sont établis des vidanges, rainures à batardeaux et déversoirs de sécurité supplémentaires, et que sont créées des pistes d'exploitation, permettant l'accès des véhicules d'intervention et de surveillance, à la place des anciennes pistes piétonnières destinées aux gardes-canaux et qui avaient été aménagées à une époque où les moyens de terrassement étaient limités.

Le second but est obtenu par la construction de prises complémentaires ainsi que par des travaux destinés à augmenter la capacité de transport du canal. On citera, en particulier, le rehaussement des berges, l'amélioration du coefficient de rugosité par la mise en place de revêtements lisses (enduit de mortier ou de béton sur des maçonneries grossières ou des parements rocheux, ou même revêtements en membrane plastique).

Il est en outre fort utile, tant pour diminuer l'importance des curages annuels que pour maintenir la capacité de transport du canal et pour préserver la qualité de ses eaux, de protéger l'ouvrage contre les chutes de matériaux ou apports de sable et terre par ruissellement. Une telle protection peut être réalisée par des fossés latéraux, des murettes de protection, ou, dans certains cas, par la couverture du canal.

\section{Troisième catégorie}

Elle comprend les réfections et les réparations des gros entretiens.
En effet, la modernisation d'un ancien canal est souvent l'ocdasion d'une révision complète de son état et d'une campagne de réparation des parties les plus touchées. Parfois même, la simple construction d'un ouvrage particulier décèlera les défaillances d'un revêtement ou d'une autre partie, dont on décidera alors la réfection.

C'est ainsi que l'on sera amené à traiter des joints, à faire des réfections d'enduits et des réparations de fissures, de béton armé dégradé, etc.

Il est à signaler que, pour centaines zones où l'ancien revêtement est par trop délabré, il peut s'avérer plus économique et plus rapide de le refaire en réalisant un cuvelage en béton légèrement armé, s'appuyant sur l'ancien revêtement.

\section{Difficultés caractéristiques}

Bien entendu, dans la pratique, la distinction entre ces diverses catégories n'est pas aussi stricte. Par exemple, pour les Extensions du Canall du Verdon, la confection d'un cuvelage en béton dans un vieux canal répondait simultanément à deux buts: réparer un ancien revêtement trop dégradé et, en même temps, réaliser une rehausse des berges pour augmenter la capacité de transport.

Par contre, quelle que soit leur nature, ces travaux de génie civil se trouvent confrontés aux mêmes difficultés, caractéristiques de ce genre d'intervention.

En premier lieu, comme dans toute réfection d'ouvrage ancien, on se heurte bien souvent à l'imprécision des documents d'archives (plans de bornage vagues, plans conformes à l'exécution inexistants, etc.).

Une autre difficulté provient des contraintes qui pèsent sur les programmes de travaux. En effet, l'importance même des canaux à moderniser fait qu'il est toujours difficile de les mettre en chômage pendant de longues périodes. De plus, afin de limiter la gêne pour les usagers, les chômages ne peuvent être effectués qu'en hiver, période de moindre consommation. Il s'ensuit donc une campagne de travaux qui doit être menée très rapidement dans les conditions climatiques les plus défavorables.

D'autre part, pour faire face à ces courts délais d'intervention, la modernisation doit être programmée sur plusieurs campagnes annuelles, et ce n'est pas l'un des moindres mérites de la régulation dynamique que de nécessiter seulement des équipements n'entraînant que peu de génie civil et dont la mise en œuvre peut être échelonnée avec beaucoup de souplesse.

Enfin, on doit signaler des obstacles d'ordre juridique, dus à la situation cadastrale imprécise des anciens canaux et aux droits de fait acquis par les usagers depuis la construction des ouvrages.

C'est ainsi que l'on se heurte, par exemple, à des difficultés d'accès pour construire un ouvrage de vannage, à des «servitudes» pour placer des gaines de câbles aux traversées des chemins ou pour trouver des exutoires lorsqu'on veut créer une vidange ou dévier les eaux de ruissellement d'amont...

Le problème d'occupation des sols se pose aussi de façon épineuse lorsqu'il s'agit d'implanter un bâtiment de commande, de créer une piste d'exploitation moderne ou de recalibrer le canal pour augmenter sa capacité. 
Dans ce dernier cas, une solution, si les lignes d'eau ie permettent, consiste à rehausser les berges du canal plutôt qu'à l'élargir.

C'est cette solution qui a été utilisée, sur les tronçons des Extensions du Canal du Verdon, par l'emploi de rehausses originales constituées de murs-bèches en béton armé, ne nécessitant ni mise en chômage du canal ni extension des emprises.

\section{Conclusions}

Il est possible d'améliorer le fonctionnement d'un canal, et de réduire le coût de son exploitation, par des travaux de modernisation et la mise en place d'une régulation bien étudiée.

Dans la plupart des cas, l'adoption de la régulation dynamique, conçue et mise au point par la Société du Canal de Provence et d'Aménagement de la Région Provençale, peut permettre de résoudire élégamment les problèmes que pose la maitrise du mouvement des eaux. On ne doit cependant pas oublier que la modernisation d'un canal ancien est une opération délicate, demandant une excellente connaissance de l'ouvrage à rénover, de ses conditions d'exploitation et qui implique, chez son mâtre d'ouvre, une réelle compétence dans les domaines de l'hydraulique et du génie civil, ainsi qu'une grande aptitude à résoudre sans délai les problèmes toujours complexes qui se présentent à chaque instant. 